\title{
Delay Games with WMSO+U Winning Conditions
}

\author{
Martin Zimmermann* \\ Reactive Systems Group, Saarland University, Germany \\ zimmermann@react.uni-saarland.de
}

\begin{abstract}
Delay games are two-player games of infinite duration in which one player may delay her moves to obtain a lookahead on her opponent's moves. We consider delay games with winning conditions expressed in weak monadic second order logic with the unbounding quantifier, which is able to express (un)boundedness properties.

We show that it is decidable whether the delaying player has a winning strategy using bounded lookahead and give a doubly-exponential upper bound on the necessary lookahead. In contrast, we show that bounded lookahead is not always sufficient to win such a game.
\end{abstract}

\section{Introduction}

Many of today's problems in computer science are no longer concerned with programs that transform data and then terminate, but with non-terminating reactive systems which have to interact with a possibly antagonistic environment for an unbounded amount of time. The framework of infinite two-player games is a powerful and flexible tool to verify and synthesize such systems. The seminal theorem of Büchi and Landweber [6] states that the winner of an infinite game on a finite arena with an $\omega$-regular winning condition can be determined and a corresponding finite-state winning strategy can be constructed effectively.

Ever since, this result was extended along different dimensions, e.g., the number of players, the type of arena, the type of winning condition, the type of interaction between the players (alternation or concurrency), zero-sum or nonzero-sum, and complete or incomplete information. In this work, we consider two of these dimensions, namely more expressive winning conditions and the possibility for one player to delay her moves.

Delay Games. In a delay game, one of the players can postpone her moves for some time, thereby obtaining a lookahead on her opponent's moves. This allows her to win some games which she loses without lookahead, e.g., if her first move depends on the third move of her opponent. Nevertheless, there are winning conditions that cannot be won with any finite lookahead, e.g., if her first move depends on every move of her opponent. Delay arises naturally when transmission of data in networks or components equipped with buffers are modeled.

* Supported by the DFG projects "TriCS" (ZI 1516/1-1) and "AVACS" (SFB/TR 14). 
From a more theoretical point of view, uniformization of relations by continuous functions 2425] can be expressed and analyzed using delay games. We consider games in which two players pick letters from alphabets $\Sigma_{I}$ and $\Sigma_{O}$, respectively, thereby producing two infinite sequences $\alpha$ and $\beta$. Thus, a strategy for the second player induces a mapping $\tau: \Sigma_{I}^{\omega} \rightarrow \Sigma_{O}^{\omega}$. It is winning for the second player if $(\alpha, \tau(\alpha))$ is contained in the winning condition $L \subseteq \Sigma_{I}^{\omega} \times \Sigma_{O}^{\omega}$ for every $\alpha$. Then, $\tau$ uniformizes $L$. In the classical setting, in which the players pick letters in alternation, the $n$-th letter of $\tau(\alpha)$ depends only on the first $n$ letters of $\alpha$. A strategy with bounded lookahead, i.e., only finitely many moves are postponed, induces a Lipschitz-continuous function $\tau$ (in the Cantor topology on $\Sigma^{\omega}$ ) and a strategy with arbitrary lookahead induces a continuous function (or equivalently, a uniformly continuous function, as $\Sigma^{\omega}$ is compact).

Hosch and Landweber proved that it is decidable whether a game with $\omega$ regular winning condition can be won with bounded lookahead [17]. This result was improved by Holtmann, Kaiser, and Thomas who showed that if a player wins a game with arbitrary lookahead, then already with doubly-exponential bounded lookahead, and gave a streamlined decidability proof yielding an algorithm with doubly-exponential running time [16. Again, these results were improved by giving an exponential upper bound on the necessary lookahead and showing ExPTIME-completeness of the solution problem [18. Going beyond $\omega$-regular winning conditions by considering context-free conditions leads to undecidability and non-elementary lower bounds on the necessary lookahead, even for very weak fragments [14].

Thus, stated in terms of uniformization, Hosch and Landweber proved decidability of the uniformization problem for $\omega$-regular relations by Lipschitzcontinuous functions and Holtmann et al. proved the equivalence of the existence of a continuous uniformization function and the existence of a Lipschitzcontinuous uniformization function for $\omega$-regular relations. Furthermore, uniformization of context-free relations is undecidable, even with respect to Lipschitz-continuous functions.

In another line of work, Carayol and Löding considered the case of finite words [8], and Löding and Winter [20] considered the case of finite trees, which are both decidable. However, Carayol and Löding showed that uniformization over infinite trees fails [7].

$W M S O+U$. In this work, we consider another class of conditions that go beyond the $\omega$-regular ones. Recall that the $\omega$-regular languages are exactly those that are definable in monadic second order logic (MSO) [5]. Recently, Bojańczyk has started a program investigating the logic $\mathrm{MSO}+\mathrm{U}$, MSO extended with the unbounding quantifier $\mathrm{U}$. A formula $\mathrm{U} X \varphi(X)$ is satisfied, if there are arbitrarily large finite sets $X$ such that $\varphi(X)$ holds. MSO $+\mathrm{U}$ is able to express all $\omega$-regular languages as well as non-regular languages like

$$
L=\left\{a^{n_{0}} b a^{n_{1}} b a^{n_{2}} b \cdots \mid \lim \sup _{i} n_{i}=\infty\right\} .
$$

Decidability of MSO+U turns out to be a delicate issue: there is no algorithm that decides $\mathrm{MSO}+\mathrm{U}$ on infinite trees and has a correctness proof using the 
axioms of ZFC [3. Unconditional undecidability on trees and decidability of $\mathrm{MSO}+\mathrm{U}$ on words are still open. However, considering weak 1$]$ MSO with the unbounding quantifier (denoted by prepending a $\mathrm{W}$ ) turned out to be promising: $\mathrm{WMSO}+\mathrm{U}$ on infinite words [1] and on infinite trees 4] and $\mathrm{WMSO}+\mathrm{U}$ with the path quantifier (WMSO+UP) on infinite trees [2] have equivalent automata models with decidable emptiness. Hence, these logics are decidable.

For $\mathrm{WMSO}+\mathrm{U}$ on infinite words, these automata are called max-automata, deterministic automata with counters whose acceptance conditions are a boolean combination of conditions "counter $c$ is bounded during the run". While processing the input, a counter may be incremented, reset to zero, or the maximum of two counters may be assigned to it (hence the name max-automata). In this work, we investigate delay games with winning conditions given by max-automata, socalled max-regular conditions.

Our Contribution. We prove the analogue of the Hosch-Landweber Theorem for max-regular winning conditions: it is decidable whether the delaying player has a winning strategy with bounded lookahead. Furthermore, we obtain a doublyexponential upper bound on the necessary lookahead, if this is the case. Finally, we present a max-regular delay game such that the delaying player wins the game, but only with unbounded lookahead. Thus, unlike for $\omega$-regular conditions, bounded lookahead is not sufficient. These are, to the best of our knowledge, the first results on delay games with quantitative winning conditions.

$\mathrm{WMSO}+\mathrm{U}$ is able to express many quantitative winning conditions studied in the literature, e.g., winning conditions in parameterized temporal logics like Prompt-LTL [19], Parametric LTL [26], or Parametric LDL [12], finitary parity and Streett games [9], and parity and Streett games with costs [13]. Thus, for all these conditions we can decide whether Player $O$ wins a delay game with bounded lookahead.

Our proof consists of a reduction to a delay-free game with a max-regular winning condition. Such games can be solved by expressing them as a satisfiability problem for WMSO+UP on infinite trees: the strategy of one player is an additional labeling of the tree and a path quantifier is able to range over all strategies of the opponent2. The reduction itself is an extension of the one used in the ExPTIME-algorithm for delay games with $\omega$-regular winning conditions [18] and is based on an equivalence relation that captures the behavior of the automaton recognizing the winning condition. However, unlike the relation used for $\omega$-regular conditions, ours is only correct if applied to words of bounded lengths. Thus, we can deal with bounded lookahead, but not with arbitrary lookahead.

\section{Definitions}

The set of non-negative integers is denoted by $\mathbb{N}$. An alphabet $\Sigma$ is a non-empty finite set of letters, and $\Sigma^{*}\left(\Sigma^{n}, \Sigma^{\omega}\right)$ denotes the set of finite words (words of

\footnotetext{
${ }^{1}$ Here, the second-order quantifiers are restricted to finite sets.

${ }^{2}$ See Example 1 in [2] for more details.
} 
length $n$, infinite words) over $\Sigma$. The empty word is denoted by $\varepsilon$, the length of a finite word $w$ by $|w|$. For $w \in \Sigma^{*} \cup \Sigma^{\omega}$ we write $w(n)$ for the $n$-th letter of $w$.

Automata. Given a finite set $C$ of counters storing non-negative integers,

$$
\operatorname{Ops}(C)=\left\{c:=c+1, c:=0, c:=\max \left(c_{0}, c_{1}\right) \mid c, c_{0}, c_{1} \in C\right\}
$$

is the set of counter operations over $C$. A counter valuation over $C$ is a mapping $\nu: C \rightarrow \mathbb{N}$. By $\nu \pi$ we denote the counter valuation that is obtained by applying a finite sequence $\pi \in \operatorname{Ops}(C)^{*}$ of counter operations to $\nu$, which is defined as implied by the operations' names.

A max-automaton $\mathcal{A}=\left(Q, C, \Sigma, q_{I}, \delta, \ell, \varphi\right)$ consists of a finite set $Q$ of states, a finite set $C$ of counters, an input alphabet $\Sigma$, an initial state $q_{I}$, a (deterministic and complete) transition function $\delta: Q \times \Sigma \rightarrow Q$, a transition labeling $\ell: \delta \rightarrow$ $\operatorname{Ops}(C)^{*}$ which labels each transition by a (possibly empty) sequence of counter operations, and an acceptance condition $\varphi$, which is a boolean formula over $C$.

A run of $\mathcal{A}$ on $\alpha \in \Sigma^{\omega}$ is an infinite sequence

$$
\rho=\left(q_{0}, \alpha(0), q_{1}\right)\left(q_{1}, \alpha(1), q_{2}\right)\left(q_{2}, \alpha(2), q_{3}\right) \cdots \in \delta^{\omega}
$$

with $q_{0}=q_{I}$. Partial (finite) runs on finite words are defined analogously, i.e., $\left(q_{0}, \alpha(0), q_{1}\right) \cdots\left(q_{n-1}, \alpha(n-1), q_{n}\right)$ is the run of $\mathcal{A}$ on $\alpha(0) \cdots \alpha(n-1)$ starting in $q_{0}$. We say that this run ends in $q_{n}$. As $\delta$ is deterministic, $\mathcal{A}$ has a unique run on every finite or infinite word.

Let $\rho$ be as in (11) and define $\pi_{n}=\ell\left(q_{n}, \alpha(n), q_{n+1}\right)$, i.e., $\pi_{n}$ is the label of the $n$-th transition of $\rho$. Given an initial counter valuation $\nu$ and a counter $c \in C$, we define the sequence

$$
\rho_{c}=\nu(c), \nu \pi_{0}(c), \nu \pi_{0} \pi_{1}(c), \nu \pi_{0} \pi_{1} \pi_{2}(c), \ldots
$$

of counter values of $c$ reached on the run after applying all operations of a transition label. A run of $\mathcal{A}$ on $\alpha$ is accepting, if the acceptance condition $\varphi$ is satisfied by the variable valuation that maps a counter $c$ to true if and only if $\lim \sup \rho_{c}$ is finite. Thus, $\varphi$ can intuitively be understood as a boolean combination of conditions "limsup $\rho_{c}<\infty$ ". Note that the limit superior of $\rho_{c}$ is independent of the initial valuation used to define $\rho_{c}$, which is the reason it is not part of the description of $\mathcal{A}$. We denote the language accepted by $\mathcal{A}$ by $L(\mathcal{A})$ and say that it is max-regular.

A parity condition (say min-parity) can be expressed in this framework using a counter for each color that is incremented every time this color is visited and employing the acceptance condition to check that the smallest color whose associated counter is unbounded, is even. Hence, the class of $\omega$-regular languages is contained in the class of max-regular languages.

Given an automaton $\mathcal{A}$ over $\Sigma_{I} \times \Sigma_{O}$, we denote by $\pi_{1}(\mathcal{A})$ the automaton obtained by projecting each letter to its first component, which recognizes the projection of $L(\mathcal{A})$ to $\Sigma_{I}$.

\footnotetext{
${ }^{3}$ Here, and later whenever convenient, we treat $\delta$ as relation $\delta \subseteq Q \times \Sigma \times Q$.
} 
Games with Delay. A delay function is a mapping $f: \mathbb{N} \rightarrow \mathbb{N} \backslash\{0\}$, which is said to be constant, if $f(i)=1$ for every $i>0$. Given a delay function $f$ and an $\omega$-language $L \subseteq\left(\Sigma_{I} \times \Sigma_{O}\right)^{\omega}$, the game $\Gamma_{f}(L)$ is played by two players (Player $I$ and Player $O$ ) in rounds $i=0,1,2, \ldots$ as follows: in round $i$, Player $I$ picks a word $u_{i} \in \Sigma_{I}^{f(i)}$, then Player $O$ picks one letter $v_{i} \in \Sigma_{O}$. We refer to the sequence $\left(u_{0}, v_{0}\right),\left(u_{1}, v_{1}\right),\left(u_{2}, v_{2}\right), \ldots$ as a play of $\Gamma_{f}(L)$, which yields two infinite words $\alpha=u_{0} u_{1} u_{2} \ldots$ and $\beta=v_{0} v_{1} v_{2} \ldots$. Player $O$ wins the play if and only if the outcome $\left(\begin{array}{l}\alpha(0) \\ \beta(0)\end{array}\right)\left(\begin{array}{c}\alpha(1) \\ \beta(1)\end{array}\right)\left(\begin{array}{l}\alpha(2) \\ \beta(2)\end{array}\right) \cdots$ is in $L$, otherwise Player $I$ wins.

Given a delay function $f$, a strategy for Player $I$ is a mapping $\tau_{I}: \Sigma_{O}^{*} \rightarrow \Sigma_{I}^{*}$ such that $\left|\tau_{I}(w)\right|=f(|w|)$, and a strategy for Player $O$ is a mapping $\tau_{O}: \Sigma_{I}^{*} \rightarrow$ $\Sigma_{O}$. Consider a play $\left(u_{0}, v_{0}\right),\left(u_{1}, v_{1}\right),\left(u_{2}, v_{2}\right), \ldots$ of $\Gamma_{f}(L)$. Such a play is consistent with $\tau_{I}$, if $u_{i}=\tau_{I}\left(v_{0} \cdots v_{i-1}\right)$ for every $i$; it is consistent with $\tau_{O}$, if $v_{i}=\tau_{O}\left(u_{0} \cdots u_{i}\right)$ for every $i$. A strategy $\tau$ for Player $p$ is winning for her, if every play that is consistent with $\tau$ is won by Player $p$. In this case, we say Player $p$ wins $\Gamma_{f}(L)$. A delay game is determined, if one of the players has a winning strategy.

Theorem 1. Delay games with max-regular winning conditions are determined.

Proof. We model a delay game $\Gamma_{f}(L(\mathcal{A}))$ as a parity game $\mathcal{G} \mathcal{G}$ with finitely many colors in a countable arena. As such games are determined [1022], so is $\Gamma_{f}(L(\mathcal{A})$ ).

A vertex of the parity game stores the round number $i \in \mathbb{N}$, an indicator $t \in$ $\{I, O\}$ which denotes whose player's turn it is, a state $q$ of $\mathcal{A}$, and the current lookahead $w \in \Sigma_{I}^{*}$. Furthermore, it stores the current counter valuation $\nu_{\text {cur }}$ and a counter valuation $\nu_{\max }$ keeping track of the maximal value that a counter has assumed thus far. Finally, for every counter $c$ there is a boolean flag $u_{c}$ that is set to true if the value $\nu_{\max }$ is updated.

The successors of a vertex of the form $\left(i, I, q, w, \nu_{\mathrm{cur}}, \nu_{\max },\left(u_{c}\right)_{c \in C}\right)$, i.e., it is Player I's turn, have the form $\left(i, O, q, w w^{\prime}, \nu_{\text {cur }}, \nu_{\max },\left(u_{c}\right)_{c \in C}\right)$ for some $w^{\prime} \in \Sigma_{I}^{f(i)}$, i.e., Player $I$ makes his move in round $i$ by picking some $w^{\prime} \in \Sigma_{I}^{f(i)}$, which is appended to the current lookahead. Dually, successors of a vertex of the form $\left(i, O, q, a w, \nu_{\mathrm{cur}}, \nu_{\max },\left(u_{c}\right)_{c \in C}\right)$ for $a \in \Sigma_{I}$ and $w \in \Sigma_{I}^{*}$, i.e., it is Player $O$ 's turn, have the form $\left(i+1, I, \delta\left(q,\left(\begin{array}{c}a \\ b\end{array}\right)\right), w, \nu_{\text {cur }}^{\prime}, \nu_{\max }^{\prime},\left(u_{c}^{\prime}\right)_{c \in C}\right)$ for some $b \in \Sigma_{O}$, where we have $\pi=\ell\left(q,\left(\begin{array}{l}a \\ b\end{array}\right), \delta\left(q,\left(\begin{array}{l}a \\ b\end{array}\right)\right)\right), \nu_{\text {cur }}^{\prime}=\nu_{\text {cur }} \pi, \nu_{\max }^{\prime}(c)=$ $\max \left(\nu_{\text {cur }}^{\prime}(c), \nu_{\max }(c)\right)$, and $u_{c}^{\prime}=1$ if and only if $\nu_{\max }^{\prime}(c)>\nu_{\max }(c)$. Here, Player $O$ makes her move in round $i$, which consists of picking a letter $b$. The state of $\mathcal{A}$, the variable valuations, and the flags are updated accordingly.

A play is winning for Player $O$, if the set of counters $c$ whose flag $u_{c}$ is set to 1 infinitely often, satisfies the winning condition $\varphi$. This is a Muller condition 5 defined on a finite set of colors, namely the powerset of the set of counters. Applying the LAR-reduction turns the Muller condition into a parity condition with finitely many colors while keeping the arena countable, which yields the parity game $\mathcal{G}$.

\footnotetext{
${ }^{4}$ See, e.g., 15 for a detailed definition of parity games.

${ }^{5}$ Again, see [15] for a formal definition.
} 
Let $\nu_{0}$ map every counter to 0 . Player $p$ has a winning strategy for the delay game $\Gamma_{f}(L(\mathcal{A}))$ if and only if she has a winning strategy for the parity game $\mathcal{G}$ from the initial vertex $\left(0, I, q_{I}, \varepsilon, \nu_{0}, \nu_{0},(0)_{c \in C}\right)$. Thus, $\Gamma_{f}(L(\mathcal{A}))$ is determined, as $\mathcal{G}$ is determined.

\section{An Equivalence Relation for Max-Automata}

Fix $\mathcal{A}=\left(Q, C, \Sigma, q_{I}, \delta, \ell, \varphi\right)$. We use notions introduced in [1 to define equivalence relations over sequences of counter operations and over words over $\Sigma$ that capture the behavior of $\mathcal{A}$.

First, we define inductively what it means for a sequence $\pi \in \operatorname{Ops}(C)^{*}$ to transfer a counter $c$ to a counter $d$. The empty sequence and the operation $c:=$ $c+1$ transfer every counter to itself. The operation $c:=0$ transfers every counter but $c$ to itself and the operation $c:=\max \left(c_{0}, c_{1}\right)$ transfers every counter but $c$ to itself and transfers $c_{0}$ and $c_{1}$ to $c$. Furthermore, if $\pi_{0}$ transfers $c$ to $e$ and $\pi_{1}$ transfers $e$ to $d$, then $\pi_{0} \pi_{1}$ transfers $c$ to $d$. If $\pi$ transfers $c$ to $d$, then we have $\nu \pi(d) \geq \nu(c)$ for every counter valuation $\nu$.

Furthermore, a sequence of counter operations $\pi$ transfers $c$ to $d$ with an increment, if there is a counter $e$ and a decomposition $\pi_{0}(e:=e+1) \pi_{1}$ of $\pi$ such that $\pi_{0}$ transfers $c$ to $e$ and $\pi_{1}$ transfers $e$ to $d$. If $\pi$ transfers $c$ to $d$ with an increment, then we have $\nu \pi(d) \geq \nu(c)+1$ for every counter valuation $\nu$.

Finally, we say that $\pi$ is a $c$-trace of length $m$, if there is a decomposition $\pi=$ $\pi_{0} \cdots \pi_{m-1}$ and a sequence of counters $c_{0}, c_{1}, \ldots, c_{m}$ with $c_{m}=c$ such that each $\pi_{i}$ transfers $c_{i}$ to $c_{i+1}$ with an increment. If $\pi$ is a $c$-trace of length $m$, then we have $\nu \pi(c) \geq m$ for every counter valuation $\nu$.

Let $\rho$ be a run and let $\pi_{i}$ be the label of the $i$-th transition of $\rho$. We say that a $c$-trace $\pi$ is contained in $\rho$, if there is an $i$ such that $\pi$ is a suffix of $\pi_{0} \cdots \pi_{i}$.

Lemma 1 ([1]). Let $\rho$ be a run of $\mathcal{A}$ and $c$ a counter. Then, $\lim \sup \rho_{c}=\infty$ if and only if $\rho$ contains arbitrarily long $c$-traces.

We use the notions of transfer (with an increment) to define the equivalence relations that capture $\mathcal{A}$ 's behavior. We say that two finite sequences of counter operations $\pi$ and $\pi^{\prime}$ are equivalent, if for all counters $c$ and $d, \pi$ transfers $c$ to $d$ if and only if $\pi^{\prime}$ transfers $c$ to $d$ and $\pi$ transfers $c$ to $d$ with an increment if and only if $\pi^{\prime}$ transfers $c$ to $d$ with an increment. We denote this equivalence relation over $\operatorname{Ops}(C)^{*}$ by $\equiv_{\mathrm{ops}}$. Using this, we define two words $x, x^{\prime} \in \Sigma^{*}$ to be equivalent, if for all states $q \in Q$, the run of $\mathcal{A}$ on $x$ starting in $q$ and the run of $\mathcal{A}$ on $x^{\prime}$ starting in $q$ end in the same state and their sequences of counter operations are $\equiv_{\mathrm{ops}^{-}}$-equivalent. We denote this equivalence over $\Sigma^{*}$ by $\equiv_{\mathcal{A}}$.

Remark 1. Let $\mathcal{A}$ be a max-automaton with $n$ states and $k$ counters.

1. The index of $\equiv_{\mathrm{ops}}$ is at most $2^{3 k^{2}}$.

2. The index of $\equiv_{\mathcal{A}}$ is at most $2^{n\left(\log n+3 k^{2}\right)}$. 
Next, we show that we can decompose an infinite word $\alpha$ into $x_{0} x_{1} x_{2} \cdots$ and replace each $x_{i}$ by an $\equiv_{\mathcal{A}}$-equivalent $x_{i}^{\prime}$ without changing membership in $L(\mathcal{A})$, provided the lengths of the $x_{i}$ and the lengths of the $x_{i}^{\prime}$ are bounded.

Lemma 2. Let $\left(x_{i}\right)_{i \in \mathbb{N}}$ and $\left(x_{i}^{\prime}\right)_{i \in \mathbb{N}}$ be two sequences of words over $\Sigma^{*}$ with $\sup _{i}\left|x_{i}\right|<\infty, \sup _{i}\left|x_{i}^{\prime}\right|<\infty$, and $x_{i} \equiv_{\mathcal{A}} x_{i}^{\prime}$ for all $i$. Then, $x=x_{0} x_{1} x_{2} \cdots \in$ $L(\mathcal{A})$ if and only if $x^{\prime}=x_{0}^{\prime} x_{1}^{\prime} x_{2}^{\prime} \cdots \in L(\mathcal{A})$.

Proof. Let $\rho$ and $\rho^{\prime}$ be the run of $\mathcal{A}$ on $x$ and $x^{\prime}$, respectively. We show that $\rho$ contains arbitrarily long $c$-traces if and only if $\rho^{\prime}$ contains arbitrarily long $c$ traces. Due to Lemma 1, this suffices to show that the run of $\mathcal{A}$ on $x$ is accepting if and only if the run of $\mathcal{A}$ on $x^{\prime}$ is accepting. Furthermore, due to symmetry, it suffices to show one direction of the equivalence. Thus, assume $\rho$ contains arbitrarily long $c$-traces and pick $m^{\prime} \in \mathbb{N}$ arbitrarily. We show the existence of a $c$-trace of length $m^{\prime}$ contained in $\rho^{\prime}$. To this end, we take a $c$-trace in $\rho$ of length $m>m^{\prime}$ for some sufficiently large $m$ and show that the $\equiv_{\mathrm{ops}}$-equivalent part of $\rho^{\prime}$ contains a $c$-trace of length $m^{\prime}$.

By definition of $\equiv_{\mathcal{A}}$, processing $x_{0} \cdots x_{i-1}$ and processing $x_{0}^{\prime} \cdots x_{i-1}^{\prime}$ brings $\mathcal{A}$ to the same state, call it $q_{i}$. Furthermore, let $\pi_{i}$ be the sequence of counter operations labeling the run of $\mathcal{A}$ on $x_{i}$ starting in $q_{i}$, which ends in $q_{i+1}$. The sequences $\pi_{i}^{\prime}$ labeling the runs on the $x_{i}^{\prime}$ are defined analogously. By $x_{i} \equiv_{\mathcal{A}} x_{i}^{\prime}$ we conclude that $\pi_{i}$ and $\pi_{i}^{\prime}$ are $\equiv_{\mathrm{ops}^{-}}$-equivalent as well. Furthermore, define $b=\sup _{i}\left|x_{i}\right|$, which is well-defined due to our assumption, and define $m=$ $\left(m^{\prime}+1\right) \cdot o \cdot b$, where $o$ is the maximal length of a sequence of operations labeling a transition, i.e., $o=\max _{\left(q, a, q^{\prime}\right) \in \delta}\left|\ell\left(q, a, q^{\prime}\right)\right|$. Each $\pi_{i}$ can contribute at most $\left|\pi_{i}\right|$ increments to a $c$-trace that subsumes $\pi_{i}$, which is bounded by $\left|\pi_{i}\right| \leq o \cdot b$.

Now, we pick $i$ such that $\pi_{0} \cdots \pi_{i}$ has a suffix that is a $c$-trace of length $m$, say the suffix starts in $\pi_{s}$. Hence, there are counters $c_{s}, c_{s+1}, \ldots, c_{i}$ such that $\pi_{j+1}$ transfers $c_{j}$ to $c_{j+1}$ for every $j$ in the range $s \leq j<i$. Furthermore, by the choice of $m$ we know that at least $m^{\prime}$ of these transfers are actually transfers with increments, as every transfer contains at most $b \cdot o$ increments.

Thus, the equivalence of $\pi_{j}$ and $\pi_{j}^{\prime}$ implies that $\pi_{j}^{\prime}$ realizes the same transfers (with increments) as $\pi_{j}$. Hence, there is a suffix of $\pi_{0}^{\prime} \cdots \pi_{i}^{\prime}$ that is a $c$-trace of length $m^{\prime}$, i.e., $\rho^{\prime}$ does contains a $c$-trace of length $m^{\prime}$.

Note that the lemma does not hold if we drop the boundedness requirements on the lengths of the $x_{i}$ and the $x_{i}^{\prime}$.

To conclude, we show that the equivalence classes of $\equiv_{\mathcal{A}}$ are regular and can be tracked on-the-fly by a finite automaton $\mathcal{T}$ in the following sense.

Lemma 3. There is a deterministic finite automaton $\mathcal{T}$ with set of states $\Sigma / \equiv_{\mathcal{A}}$ such that the run of $\mathcal{T}$ on $w \in \Sigma^{*}$ ends in $[w]_{\equiv_{\mathcal{A}}}$.

Proof. Define $\mathcal{T}=\left(\Sigma / \equiv_{\mathcal{A}}, \Sigma,[\varepsilon]_{\equiv_{\mathcal{A}}}, \delta_{\mathcal{T}}, \emptyset\right)$ where $\delta_{\mathcal{T}}\left([x]_{\equiv_{\mathcal{A}}}, a\right)=[x a]_{\equiv_{\mathcal{A}}}$, which is independent of the representative $x$ and based on the fact that $\equiv_{\mathrm{ops}}$ (and thus also $\left.\equiv_{\mathcal{A}}\right)$ is a congruence, i.e., $\pi_{0} \equiv_{\mathrm{ops}} \pi_{1}$ implies $\pi_{0} \pi \equiv_{\mathrm{ops}} \pi_{1} \pi$ for every $\pi$. A straightforward induction over $|w|$ shows that $\mathcal{T}$ has the desired properties.

Corollary 1. Every $\equiv_{\mathcal{A}}$-equivalence class is regular. 


\section{Reducing Delay Games to Delay-free Games}

In this section, we prove our main theorem.

Theorem 2. The following problem is decidable: given a max-automaton $\mathcal{A}$, does Player $O$ win $\Gamma_{f}(L(\mathcal{A}))$ for some constant delay function $f$ ?

To prove this result, we construct a delay-free game in a finite arena with a max-regular winning condition that is won by Player $O$ if and only if she wins $\Gamma_{f}(L(\mathcal{A}))$ for some constant delay function $f$. The winner of such a game can be determined effectively.

Let $\mathcal{A}=\left(Q, C, \Sigma_{I} \times \Sigma_{O}, q_{I}, \delta, \ell, \varphi\right)$ and let $\mathcal{T}=\left(\left(\Sigma_{I} \times \Sigma_{O}\right) / \equiv_{\mathcal{A}}, \Sigma_{I} \times\right.$ $\left.\Sigma_{O},[\varepsilon]_{\equiv \mathcal{A}}, \delta_{\mathcal{T}}, \emptyset\right)$ be defined as in Lemma 3. For the sake of readability, we denote

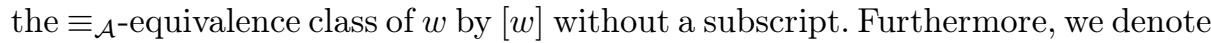
equivalence classes using the letter $S$. We define the product $\mathcal{P}=\left(Q_{\mathcal{P}}, C, \Sigma_{I} \times\right.$ $\left.\Sigma_{O}, q_{I}^{\mathcal{P}}, \delta_{\mathcal{P}}, \ell_{\mathcal{P}}, \varphi\right)$ of $\mathcal{A}$ and $\mathcal{T}$, which is a max-automaton, where

$-Q_{\mathcal{P}}=Q \times\left(\left(\Sigma_{I} \times \Sigma_{O}\right) / \equiv_{\mathcal{A}}\right)$,

$-q_{I}^{\mathcal{P}}=\left(q_{I},[\varepsilon]_{\equiv \mathcal{A}}\right)$,

- $\delta_{\mathcal{P}}((q, S), a)=\left(\delta(q, a), \delta_{\mathcal{T}}(S, a)\right)$ for a states $q \in Q$, an equivalence class $S \in$ $\left(\Sigma_{I} \times \Sigma_{O}\right) / \equiv_{\mathcal{A}}$, and a letter $a \in \Sigma_{I} \times \Sigma_{O}$, and

$-\ell_{\mathcal{P}}\left((q, S), a,\left(q^{\prime}, S^{\prime}\right)\right)=\ell\left(q, a, q^{\prime}\right)$.

Let $n=\left|Q_{\mathcal{P}}\right|$. We have $L(\mathcal{P})=L(\mathcal{A})$, since acceptance only depends on the component $\mathcal{A}$ of $\mathcal{P}$. However, we are interested in partial runs of $\mathcal{P}$, as the component $\mathcal{T}$ keeps track of the equivalence class of the input processed by $\mathcal{P}$.

Remark 2. Let $w \in\left(\Sigma_{I} \times \Sigma_{O}\right)^{*}$ and let $\left(q_{0}, S_{0}\right)\left(q_{1}, S_{1}\right) \cdots\left(q_{|w|}, S_{|w|}\right)$ be the run of $\mathcal{P}$ on $w$ from some state $\left(q_{0}, S_{0}\right)$ with $S_{0}=[\varepsilon]$. Then, $q_{0} q_{1} \cdots q_{|w|}$ is the run of $\mathcal{A}$ on $w$ starting in $q_{0}$ and $S_{|w|}=[w]$.

In the following, we will work with partial functions $r$ from $Q_{\mathcal{P}}$ to $2^{Q_{\mathcal{P}}}$, where we denote the domain of $r$ by $\operatorname{dom}(r)$. Intuitively, we use such a function to capture the information encoded in the lookahead provided by Player $I$. Assume Player $I$ has picked $\alpha(0) \cdots \alpha(j)$ and Player $O$ has picked $\beta(0) \cdots \beta(i)$ for some $i<j$, i.e., the lookahead is $\alpha(i+1) \cdots \alpha(j)$. Then, we can determine the state $q$ that $\mathcal{P}$ reaches when processing $\left(\begin{array}{c}\alpha(0) \\ \beta(0)\end{array}\right) \cdots\left(\begin{array}{c}\alpha(i) \\ \beta(i)\end{array}\right)$, but the automaton cannot process $\alpha(i+1) \cdots \alpha(j)$, since Player $O$ has not yet provided her moves $\beta(i+1) \cdots \beta(j)$. However, we can determine which states Player $O$ can enforce by picking an appropriate completion. These will be contained in $r(q)$.

To formalize this, we use the function $\delta_{\text {pow }}: 2^{Q_{\mathcal{P}}} \times \Sigma_{I} \rightarrow 2^{Q_{\mathcal{P}}}$ defined via $\delta_{\text {pow }}(P, a)=\bigcup_{q \in P} \bigcup_{b \in \Sigma_{O}} \delta_{\mathcal{P}}\left(q,\left(\begin{array}{l}a \\ b\end{array}\right)\right)$, i.e., $\delta_{\text {pow }}$ is the transition function of the powerset automaton of the projection automaton $\pi_{1}(\mathcal{P})$. As usual, we extend $\delta_{\text {pow }}$ to $\delta_{\text {pow }}^{*}: 2^{Q_{\mathcal{P}}} \times \Sigma_{I}^{*} \rightarrow 2^{Q_{\mathcal{P}}}$ via $\delta_{\text {pow }}^{*}(P, \varepsilon)=P$ and $\delta_{\text {pow }}^{*}(P, w a)=$ $\delta_{\text {pow }}\left(\delta_{\text {pow }}^{*}(P, w), a\right)$.

Let $D \subseteq Q_{\mathcal{P}}$ be non-empty and let $w \in \Sigma_{I}^{*}$. We define the function $r_{w}^{D}$ with domain $D$ as follows: for every $(q, S) \in D$, we have

$$
r_{w}^{D}(q, S)=\delta_{\text {pow }}^{*}(\{(q,[\varepsilon])\}, w)
$$


i.e., we collect all states $\left(q^{\prime}, S^{\prime}\right)$ reachable from $(q,[\varepsilon]$ ) (note that the second component is the equivalence class of the empty word, not the class $S$ from the argument) via a run of $\pi_{1}(\mathcal{P})$ on $w$. Thus, if $\left(q^{\prime}, S^{\prime}\right) \in r_{w}^{D}(q, S)$, then there is a word $w^{\prime}$ whose projection is $w$ and with $\left[w^{\prime}\right]=S^{\prime}$ such that the run of $\mathcal{A}$ on $w^{\prime}$ leads from $q$ to $q^{\prime}$. Thus, if Player $I$ has picked the lookahead $w$, then Player $O$ could pick an answer such that the combined word leads $\mathcal{A}$ from $q$ to $q^{\prime}$ and such that it is a representative of $S^{\prime}$.

We call $w$ a witness for a partial function $r: Q_{\mathcal{P}} \rightarrow 2^{Q_{\mathcal{P}}}$, if we have $r=$ $r_{w}^{\operatorname{dom}(r)}$. Thus, we obtain a language $W_{r} \subseteq \Sigma_{I}^{*}$ of witnesses for each such function $r$. Now, we define $\mathfrak{R}=\left\{r \mid \operatorname{dom}(r) \neq \emptyset\right.$ and $W_{r}$ is infinite $\}$.

Lemma 4. Let $\mathfrak{R}$ be defined as above.

1. Let $r \in \Re$. Then, $r(q) \neq \emptyset$ for every $q \in \operatorname{dom}(r)$.

2. Let $r$ be a partial function from $Q_{\mathcal{P}}$ to $2^{Q_{\mathcal{P}}}$. Then, $W_{r}$ is recognized by a deterministic finite automaton with $2^{n^{2}}$ states.

3. Let $r \in \mathfrak{R}$. Then, $W_{r}$ contains a word $w$ with $k \leq|w| \leq k+2^{n^{2}}$ for every $k$.

4. Let $r \neq r^{\prime} \in \mathfrak{R}$ such that $\operatorname{dom}(r)=\operatorname{dom}\left(r^{\prime}\right)$. Then, $W_{r} \cap W_{r^{\prime}}=\emptyset$.

5. Let $D \subseteq Q_{\mathcal{P}}$ be non-empty and let $w$ be such that $|w| \geq 2^{n^{2}}$. Then, there exists some $r \in \mathfrak{R}$ with $\operatorname{dom}(r)=D$ and $w \in W_{r}$.

Due to items 4) and [5.), we can define for every non-empty $D \subseteq Q_{\mathcal{P}}$ a function $r_{D}$ that maps words $w \in \Sigma_{I}^{*}$ with $|w| \geq 2^{n^{2}}$ to the unique function $r$ with $\operatorname{dom}(r)=D$ and $w \in W_{r}$. This will be used later in the proof.

Now, we define an abstract game $\mathcal{G}(\mathcal{A})$ between Player $I$ and Player $O$ that is played in rounds $i=0,1,2, \ldots$ in each round, Player $I$ picks a function from $\mathfrak{R}$ and then Player $O$ picks a state $q$ of $\mathcal{P}$. In round 0 , Player $I$ has to pick $r_{0}$ subject to constraint $(\mathrm{C} 1): \operatorname{dom}\left(r_{0}\right)=\left\{q_{I}^{\mathcal{P}}\right\}$. Then, Player $O$ has to pick a state $q_{0} \in$ $\operatorname{dom}\left(r_{0}\right)$ (which implies $q_{0}=q_{I}^{\mathcal{P}}$ ). Now, consider round $i>0$ : Player $I$ has picked functions $r_{0}, r_{1}, \ldots, r_{i-1}$ and Player $O$ has picked states $q_{0}, q_{1}, \ldots, q_{i-1}$. Now, Player $I$ has to pick a function $r_{i}$ subject to constraint $(\mathrm{C} 2): \operatorname{dom}\left(r_{i}\right)=$ $r_{i-1}\left(q_{i-1}\right)$. Then, Player $O$ has to pick a state $q_{i} \in \operatorname{dom}\left(r_{i}\right)$. Both players can always move: Player $I$ can, as $r_{i-1}\left(q_{i-1}\right)$ is always non-empty (Lemma 4II) and thus the domain of some $r \in \mathfrak{R}$ (Lemma 45) and Player $O$ can, as the domain of every $r \in \mathfrak{R}$ is non-empty by construction.

The resulting play is the sequence $r_{0} q_{0} r_{1} q_{1} r_{2} q_{2} \cdots$. Let $q_{i}=\left(q_{i}^{\prime}, S_{i}\right)$ for every $i$, i.e., $S_{i}$ is an $\equiv_{\mathcal{A}}$-equivalence class. Let $x_{i} \in S_{i}$ for every $i$ such that $\sup _{i}\left|x_{i}\right|<$ $\infty$. Such a sequence can always be found as $\equiv_{\mathcal{A}}$ has finite index. Player $O$ wins the play if the word $x_{0} x_{1} x_{2} \ldots$ is accepted by $\mathcal{A}$. Due to Lemma 2 this definition is independent of the choice of the representatives $x_{i}$.

A strategy for Player $I$ is a function $\tau_{I}^{\prime}$ mapping the empty play prefix to a function $r_{0}$ subject to constraint $(\mathrm{C} 1)$ and mapping a non-empty play prefix $r_{0} q_{0} \cdots r_{i-1} q_{i-1}$ ending in a state to a function $r_{i}$ subject to constraint (C2). On the other hand, a strategy for Player $O$ maps a play prefix $r_{0} q_{0} \cdots r_{i}$ ending in a function to a state $q_{i} \in \operatorname{dom}\left(r_{i}\right)$. A play $r_{0} q_{0} r_{1} q_{1} r_{2} q_{2} \ldots$ is consistent with $\tau_{I}^{\prime}$, if $r_{i}=\tau_{I}^{\prime}\left(r_{0} q_{0} \cdots r_{i-1} q_{i-1}\right)$ for every $i \geq 0$. Dually, the play is consistent with 
$\tau_{O}^{\prime}$, if $q_{i}=\tau_{O}^{\prime}\left(r_{0} q_{0} \cdots r_{i}\right)$ for every $i \geq 0$. A strategy is winning for Player $p$, if every play that is consistent with this strategy is winning for her. As usual, we say that Player $p$ wins $\mathcal{G}(\mathcal{A})$, if she has a winning strategy.

Lemma 5. Player $O$ wins $\Gamma_{f}(L(\mathcal{A}))$ for some constant delay function $f$ if and only if Player $O$ wins $\mathcal{G}(\mathcal{A})$.

Proof. First, assume Player $O$ has a winning strategy $\tau_{O}$ for $\Gamma_{f}(L(\mathcal{A}))$ for some constant delay function $f$. We construct a winning strategy $\tau_{O}^{\prime}$ for Player $O$ in $\mathcal{G}(\mathcal{A})$ via simulating a play of $\mathcal{G}(\mathcal{A})$ by a play of $\Gamma_{f}(L(\mathcal{A}))$.

Let $r_{0}$ be the first move of Player $I$ in $\mathcal{G}(\mathcal{A})$, which has to be responded to by Player $O$ by picking $q_{I}^{\mathcal{P}}=\tau_{O}^{\prime}\left(r_{0}\right)$, and let $r_{1}$ be Player $I$ 's response to that move. Let $w_{0} \in W_{r_{0}}$ and $w_{1} \in W_{r_{1}}$ be witnesses for the functions picked by Player $I$. Due to Lemma 413, we can choose $w_{0}$ and $\left|w_{1}\right|$ with $f(0) \leq\left|w_{0}\right|,\left|w_{1}\right| \leq f(0)+2^{n^{2}}$.

We simulate the play prefix $r_{0} q_{0} r_{1}$ in $\Gamma_{f}\left(L(\mathcal{A})\right.$ ), where $q_{0}=q_{I}^{\mathcal{P}}$ : Player $I$ picks $w_{0} w_{1}=\alpha(0) \cdots \alpha\left(\ell_{1}-1\right)$ in his first moves and let $\beta(0) \cdots \beta\left(\ell_{1}-f(0)\right)$ be the response of Player $O$ according to $\tau_{O}$. We obtain $\left|\beta(0) \cdots \beta\left(\ell_{1}-f(0)\right)\right| \geq\left|w_{0}\right|$, as $\left|w_{1}\right| \geq f(0)$.

Thus, we are in the following situation for $i=1$ : in $\mathcal{G}(\mathcal{A})$, we have a play prefix $r_{0} q_{0} \cdots r_{i-1} q_{i-1} r_{i}$ and in $\Gamma_{f}(L(\mathcal{A}))$, Player $I$ has picked $w_{0} w_{1} \cdots w_{i}=$ $\alpha(0) \cdots \alpha\left(\ell_{i}-1\right)$ and Player $O$ has picked $\beta(0) \cdots \beta\left(\ell_{i}-f(0)\right)$ according to $\tau_{O}$, where $\left|\beta(0) \cdots \beta\left(\ell_{i}-f(0)\right)\right| \geq\left|w_{0} \cdots w_{i-1}\right|$. Furthermore, $w_{j}$ is a witness for $r_{j}$ for every $j \leq i$.

In this situation, let $q_{i}$ be the state of $\mathcal{P}$ that is reached when processing $w_{i-1}$ and the corresponding moves of Player $O$, i.e.,

$$
\left(\begin{array}{c}
\alpha\left(\left|w_{0} \cdots w_{i-2}\right|\right) \\
\beta\left(\left|w_{0} \cdots w_{i-2}\right|\right)
\end{array}\right) \cdots\left(\begin{array}{c}
\alpha\left(\left|w_{0} \cdots w_{i-1}\right|-1\right) \\
\beta\left(\left|w_{0} \cdots w_{i-1}\right|-1\right)
\end{array}\right)
$$

starting in state $\left(q_{i-1}^{\prime},[\varepsilon]\right)$, where $q_{i-1}=\left(q_{i-1}^{\prime}, S_{i-1}\right)$.

By definition of $r_{i-1}$, we have $q_{i} \in r_{i-1}\left(q_{i-1}\right)$, i.e., $q_{i}$ is a legal move for Player $O$ in $\mathcal{G}(\mathcal{A})$ to extend the play prefix $r_{0} q_{0} \cdots r_{i-1} q_{i-1} r_{i}$. Thus, we define $\tau_{O}^{\prime}\left(r_{0} q_{0} \cdots r_{i-1} q_{i-1} r_{i}\right)=q_{i}$. Now, let $r_{i+1}$ be the next move of Player $I$ in $\mathcal{G}(\mathcal{A})$ and let $w_{i+1} \in W_{r_{i+1}}$ be a witness with $f(0) \leq\left|w_{i+1}\right| \leq f(0)+2^{n^{2}}$. Going back to $\Gamma_{f}(L(\mathcal{A}))$, let Player $I$ pick $w_{i+1}=\alpha\left(\ell_{i}\right) \cdots \alpha\left(\ell_{i+1}-1\right)$ as his next moves and let $\beta\left(\ell_{i}-f(0)+1\right) \cdots \beta\left(\ell_{i+1}-f(0)\right)$ be the response of Player $O$ according to $\tau_{O}$. Then, we are in the situation as described in the previous paragraph, which concludes the definition of $\tau_{O}^{\prime}$.

It remains to show that $\tau_{O}^{\prime}$ is winning for Player $O$ in $\mathcal{G}(\mathcal{A})$. Consider a play $r_{0} q_{0} r_{1} q_{1} r_{2} q_{2} \cdots$ that is consistent with $\tau_{O}^{\prime}$ and let $w=\left(\begin{array}{c}\alpha(0) \\ \beta(0)\end{array}\right)\left(\begin{array}{l}\alpha(1) \\ \beta(1)\end{array}\right)\left(\begin{array}{l}\alpha(2) \\ \beta(2)\end{array}\right) \cdots$ be the corresponding outcome constructed as in the simulation described above. Let $q_{i}=\left(q_{i}^{\prime}, S_{i}\right)$, i.e., $q_{i}^{\prime}$ is a state of our original automaton $\mathcal{A}$. A straightforward inductive application of Remark 2 shows that $q_{i}^{\prime}$ is the state that $\mathcal{A}$ reaches after processing $w_{i}$ and the corresponding moves of Player $O$, i.e.,

$$
x_{i}=\left(\begin{array}{c}
\alpha\left(\left|w_{0} \cdots w_{i-1}\right|\right) \\
\beta\left(\left|w_{0} \cdots w_{i-1}\right|\right)
\end{array}\right) \cdots\left(\begin{array}{c}
\alpha\left(\left|w_{0} \cdots w_{i}\right|-1\right) \\
\beta\left(\left|w_{0} \cdots w_{i}\right|-1\right)
\end{array}\right),
$$


starting in $q_{i-1}$ and that $S_{i}=\left[x_{i}\right]$. Note that the length of the $x_{i}$ is bounded, i.e., we have $\sup _{i}\left|x_{i}\right| \leq f(0)+2^{n^{2}}$.

As $w$ is consistent with a winning strategy for Player $O$, the run of $\mathcal{A}$ on $w=x_{0} x_{1} x_{2} \cdots$ is accepting. Thus, we conclude that the play $r_{0} q_{0} r_{1} q_{1} r_{2} q_{2} \ldots$ is winning for Player $O$, as the $x_{i}$ are a bounded sequence of representatives. Hence, $\tau_{O}^{\prime}$ is indeed a winning strategy for Player $O$ in $\mathcal{G}(\mathcal{A})$.

Now, we consider the other implication: assume Player $O$ has a winning strategy $\tau_{O}^{\prime}$ for $\mathcal{G}(\mathcal{A})$ and fix $d=2^{n^{2}}$. We construct a winning strategy $\tau_{O}$ for her in $\Gamma_{f}(L(\mathcal{A}))$ for the constant delay function $f$ with $f(0)=2 d$. In the following, both players pick their moves in blocks of length $d$. We denote Player I's blocks by $a_{i}$ and Player $O$ 's blocks by $b_{i}$, i.e., in the following, every $a_{i}$ is in $\Sigma_{I}^{d}$ and every $b_{i}$ is in $\Sigma_{O}^{d}$. Again, we simulate a play of $\Gamma_{f}(L(\mathcal{A}))$ by a play in $\mathcal{G}(\mathcal{A})$.

Let $a_{0} a_{1}$ be the first move of Player $I$ in $\Gamma_{f}(L(\mathcal{A}))$, let $q_{0}=q_{I}^{\mathcal{P}}$, and define the functions $r_{0}=r_{\left\{q_{0}\right\}}\left(a_{0}\right)$ and $r_{1}=r_{r_{0}\left(q_{0}\right)}\left(a_{1}\right)$. Then, $r_{0} q_{0} r_{1}$ is a legal play prefix of $\mathcal{G}(\mathcal{A})$ that is consistent with the winning strategy $\tau_{O}^{\prime}$ for Player $O$.

Thus, we are in the following situation for $i=1$ : in $\mathcal{G}(\mathcal{A})$, we have constructed a play prefix $r_{0} q_{0} \cdots r_{i-1} q_{i-1} r_{i}$ that is consistent with $\tau_{O}^{\prime}$; in $\Gamma_{f}(L(\mathcal{A})$ ), Player $I$ has picked $a_{0} \cdots a_{i}$ such that $a_{j}$ is a witness for $r_{j}$ for every $j$ in the range $0 \leq$ $j \leq i$. Player $O$ has picked $b_{0} \cdots b_{i-2}$, which is the empty word for $i=1$.

In this situation, let $q_{i}=\tau_{O}^{\prime}\left(r_{0} q_{0} \cdots r_{i-1} q_{i-1} r_{i}\right)$. By definition, we have $q_{i} \in \operatorname{dom}\left(r_{i}\right)=r_{i-1}\left(q_{i-1}\right)$. Furthermore, as $a_{i-1}$ is a witness for $r_{i-1}$, there exists $b_{i-1}$ such that $\mathcal{P}$ reaches the state $q_{i}$ when processing $\left(\begin{array}{c}a_{i-1} \\ b_{i-1}\end{array}\right)$ starting in state $\left(q_{i-1}^{\prime},[\varepsilon]\right)$, where $q_{i-1}=\left(q_{i-1}^{\prime}, S_{i-1}\right)$.

Player $O$ 's strategy for $\Gamma_{f}(L(\mathcal{A}))$ is to play $b_{i-1}$ in the next $d$ rounds, which is answered by Player $I$ by picking some $a_{i+1}$ during these rounds. This induces the function $r_{i+1}=r_{r_{i}\left(q_{i}\right)}\left(a_{i+1}\right)$. Now, we are in the same situation as described in the previous paragraph. This finishes the description of the strategy $\tau_{O}$ for Player $O$ in $\Gamma_{f}(L(\mathcal{A}))$.

It remains to show that $\tau_{O}$ is winning for Player $O$. Let $w=\left(\begin{array}{c}a_{0} \\ b_{0}\end{array}\right)\left(\begin{array}{l}a_{1} \\ b_{1}\end{array}\right)\left(\begin{array}{l}a_{2} \\ b_{2}\end{array}\right) \cdots$ be the outcome of a play in $\Gamma_{f}(L(\mathcal{A}))$ that is consistent with $\tau_{O}$. Furthermore, let $r_{0} q_{0} r_{1} q_{1} r_{2} q_{2} \cdots$ be the corresponding play in $\mathcal{G}(\mathcal{A})$ constructed in the simulation as described above, which is consistent with $\tau_{O}^{\prime}$. Let $q_{i}=\left(q_{i}^{\prime}, S_{i}\right)$. A straightforward inductive application of Remark 2 shows that $q_{i}$ is the state reached by $\mathcal{A}$ after processing $x_{i}=\left(\begin{array}{c}a_{i} \\ b_{i}\end{array}\right)$ starting in $q_{i-1}$ and $S_{i}=\left[x_{i}\right]$. Furthermore, we have $\sup _{i}\left|x_{i}\right|=d$.

As $r_{0} q_{0} r_{1} q_{1} r_{2} q_{2} \ldots$ is consistent with a winning strategy for Player $O$ and therefore winning for Player $O$, we conclude that $x_{0} x_{1} x_{2} \cdots$ is accepted by $\mathcal{A}$. Hence, $\mathcal{A}$ accepts the outcome $w$, which is equal to $x_{0} x_{1} x_{2} \cdots$, i.e., the play in $\Gamma_{f}(L(\mathcal{A}))$ is winning for Player $O$. Thus, $\tau_{O}$ is a winning strategy for Player $O$ in $\Gamma_{f}(L(\mathcal{A}))$.

Now, we can prove our main theorem of this section, Theorem 2 ,

Proof. Due to Lemma 5, we just have to show that we can construct and solve an explicit version of $\mathcal{G}(\mathcal{A})$. To this end, we encode $\mathcal{G}(\mathcal{A})$ as a graph-based game with arena $\left(V, V_{I}, V_{O}, E\right)$ where 
- the set of vertices is $V=V_{I} \cup V_{O}$ with

- the vertices $V_{I}=\left\{v_{I}\right\} \cup \mathfrak{R} \times Q_{\mathcal{P}}$ of Player $I$, where $v_{I}$ is a fresh initial vertex,

- the vertices $V_{O}=\mathfrak{R}$ of Player $O$, and

- $E$ is the union of the following sets of edges:

- $\left\{\left(v_{I}, r\right) \mid \operatorname{dom}(r)=\left\{q_{I}^{\mathcal{P}}\right\}\right\}$ : the initial moves of Player $I$.

- $\left\{\left((r, q), r^{\prime}\right) \mid \operatorname{dom}\left(r^{\prime}\right)=r(q)\right\}$ : (regular) moves of Player $I$.

- $\{(r,(r, q)) \mid q \in \operatorname{dom}(r)\}$ : moves of Player $O$.

A play is an infinite path starting in $v_{I}$. To determine the winner of a play, we fix an arbitrary function rep: $\left(\Sigma_{I} \times \Sigma_{O}\right)^{*} / \equiv_{\mathcal{A}} \rightarrow\left(\Sigma_{I} \times \Sigma_{O}\right)^{*}$ that maps each equivalence class to some representative, i.e., $\operatorname{rep}(S) \in S$ for every $S \in$ $\left(\Sigma_{I} \times \Sigma_{O}\right)^{*} / \equiv_{\mathcal{A}}$. Consider an infinite play

$$
v_{I}, r_{0},\left(r_{0}, q_{0}\right), r_{1},\left(r_{1}, q_{1}\right), r_{2},\left(r_{2}, q_{2}\right), \ldots,
$$

with $q_{i}=\left(q_{i}^{\prime}, S_{i}\right)$ for every $i$. This play is winning for Player $O$, if the infinite word $\operatorname{rep}\left(S_{0}\right) \operatorname{rep}\left(S_{1}\right) \operatorname{rep}\left(S_{2}\right) \cdots$ is accepted by $\mathcal{A}\left(\right.$ note that $\sup _{i}\left|\operatorname{rep}\left(S_{i}\right)\right|$ is bounded, as there are only finitely many equivalence classes). The set Win $\subseteq V^{\omega}$ of winning plays for Player $O$ is a max-regular languag 6 , as it can be recognized by an automaton that simulates the run of $\mathcal{A}$ on $\operatorname{rep}(S)$ when processing a vertex of the form $(r,(q, S))$ and ignores all other vertices. Games in finite arenas with max-regular winning condition are decidable via an encoding as a satisfiability problem for $\mathrm{WMSO}+\mathrm{UP}[2]$.

Player $O$ wins $\mathcal{G}(\mathcal{A})$ (and thus $\Gamma_{f}(L(\mathcal{A})$ ) for some constant $f$ ) if and only if she has a winning strategy from $v_{I}$ in the game $\left(\left(V, V_{I}, V_{O}, E\right)\right.$, Win), which concludes the proof.

We obtain a doubly-exponential upper bound on the constant delay necessary for Player $O$ to win a delay game with a max-regular winning condition by applying both directions of the equivalence between $\Gamma_{f}(\mathcal{A})$ and $\mathcal{G}(\mathcal{A})$.

Corollary 2. Let $\mathcal{A}$ be a max-automaton with $n$ states and $k$ counters. The following are equivalent:

1. Player $O$ wins $\Gamma_{f}(L(\mathcal{A}))$ for some constant delay function $f$.

2. Player $O$ wins $\Gamma_{f}(L(\mathcal{A}))$ for some constant delay function $f$ with

$$
f(0) \leq 2^{2^{2 n\left(\log n+3 k^{2}\right)}+1} .
$$

\section{Constant Delay Does Not Suffice}

In this section, we show that constant delay does not suffice to win every delay game that Player $O$ can with with arbitrary delay, i.e., the analogue of the Holtmann-Kaiser-Thomas theorem for delay games with max-regular winning conditions does not hold. In terms of uniformization, the following theorem shows that there are max-regular languages that are uniformizable by (uniformly) continuous functions, but not by Lipschitz-continuous functions.

\footnotetext{
${ }^{6}$ This implies that $\mathcal{G}(\mathcal{A})$ is determined, as max-regular conditions are Borel [121.
} 
Theorem 3. There is a max-regular language $L$ such that Player $O$ wins $\Gamma_{f}(L)$ for some $f$, but not for any constant $f$.

Proof. Let $\Sigma_{I}=\{0,1, \#\}$ and $\Sigma_{O}=\{0,1, *\}$. An input block is a word \#w with $w \in\{0,1\}^{+}$, and its length is defined to be $|w|$. An output block is a word

$$
\left(\begin{array}{c}
\# \\
b
\end{array}\right)\left(\begin{array}{c}
\alpha(1) \\
*
\end{array}\right)\left(\begin{array}{c}
\alpha(2) \\
*
\end{array}\right) \cdots\left(\begin{array}{c}
\alpha(n) \\
*
\end{array}\right)\left(\begin{array}{l}
b \\
b
\end{array}\right) \in\left(\Sigma_{I} \times \Sigma_{O}\right)^{+}
$$

for $b \in\{0,1\}$ and $\alpha(j) \in\{0,1\}$ for all $j$ in the range $1 \leq j \leq n$. The length of the block is $n+1$. Note that the first and last letter in an output block are the only ones whose second component is not a $*$, and that these letters have to be equal to the first component of the last letter of the block. Every output block of length $n$ can be extended to an output block of length $n$ and and the projection to the first component of every output block is an input block.

A word $\left(\begin{array}{c}\alpha(0) \\ \beta(0)\end{array}\right)\left(\begin{array}{c}\alpha(1) \\ \beta(1)\end{array}\right)\left(\begin{array}{c}\alpha(2) \\ \beta(2)\end{array}\right) \cdots$ over $\left(\Sigma_{I} \times \Sigma_{O}\right)^{\omega}$ is in $L$ if and only if it satisfies the following property: if $\alpha(0) \alpha(1) \alpha(2) \cdots$ contains infinitely many \# and arbitrarily long input blocks, then $\left(\begin{array}{c}\alpha(0) \\ \beta(0)\end{array}\right)\left(\begin{array}{c}\alpha(1) \\ \beta(1)\end{array}\right)\left(\begin{array}{c}\alpha(2) \\ \beta(2)\end{array}\right) \cdots$ contains arbitrarily long output blocks. It is easy to come up with a WMSO+U formula defining $L$ by formalizing the definitions of input and output blocks in first-order logic.

Now, consider $L$ as winning condition for a delay game. Intuitively, Player $O$ has to specify arbitrarily long output blocks, provided Player $I$ produces arbitrarily long input blocks. The challenge for Player $O$ is that she has to specify at the beginning of every output block whether she ends the block in a position where Player $O$ has picked a 0 or a 1.

First, we show that Player $O$ wins $\Gamma_{f}(L)$ for the delay function $f$ with $f(i)=$ 2 for every $i$. Consider round $i$ for some $i$ with $\alpha(i)=\#$. By the choice of $f$, Player $O$ has already picked $\alpha(i+1) \cdots \alpha(2 i+1)$. Let $j$ in the range $i+1 \leq j \leq$ $2 i+1$ be maximal with $\alpha(i+1) \cdots \alpha(j) \in\{0,1\}^{+}$. If $j$ is defined, then Player $O$ picks $\alpha(j)$ in round $i, *$ during rounds $i+1, \ldots, j-1$, and $\alpha(j)$ in round $j$. In every other situation, she picks an arbitrary letter.

Now, consider an outcome $\left(\begin{array}{l}\alpha(0) \\ \beta(0)\end{array}\right)\left(\begin{array}{c}\alpha(1) \\ \beta(1)\end{array}\right)\left(\begin{array}{c}\alpha(2) \\ \beta(2)\end{array}\right) \cdots$ that is consistent with this strategy that contains infinitely many \# and arbitrarily long input blocks. Let $n \in \mathbb{N}$ be arbitrary and pick an input block of length at least $n$. We can assume w.l.o.g. that the block begins at $\alpha(i)$ with $i>n$. Thus, in round $i$ when Player $O$ had to pick $\beta(i)$, Player $I$ had already picked $\alpha(i+1) \cdots \alpha(2 i+1)$, which comprises the whole input block of length $n$. Accordingly, Player $O$ produces an output block of length at least $n$. Thus, the winning condition is satisfied.

It remains to show that Player $I$ wins $\Gamma_{f}(L)$ for every constant delay function $f$. He uses a counter $c$ to produce arbitrarily long input blocks, which is initialized to $f(0)$. In round 0 , he picks $\# 0^{f(0)-1}$. If Player $O$ answers the \# with 0 , then Player $I$ continues picking 1 until he has produced an input block of length $c$. Dually, if Player $O$ answers the \# with 1 or $*$, then Player $I$ continues picking 0 until he has produced an input block of length $c$. In both cases, he continues by incrementing the counter and by picking $\# 0^{f(0)-1}$ during the next rounds until Player $O$ has to respond to the last \#. Then, he continues as in the case distinction described above. 
Now, consider an outcome $\left(\begin{array}{c}\alpha(0) \\ \beta(0)\end{array}\right)\left(\begin{array}{l}\alpha(1) \\ \beta(1)\end{array}\right)\left(\begin{array}{c}\alpha(2) \\ \beta(2)\end{array}\right) \cdots$ that is consistent with this strategy. It contains infinitely many \# and arbitrarily long input blocks. Furthermore, the length of every output block is bounded by $f(0)$, as Player $I$ is able to react to Player $O$ 's declaration at the beginning of each such block by playing the opposite letter. Thus, the play is winning for Player $I$.

We have just shown that Player $O$ wins the game for the delay function $f(i)=2$. Such a function is called linear [14, as the lookahead grows linearly.

\section{Conclusion}

We considered delay games with max-regular winning conditions. Our main result is an algorithm that determines whether Player $O$ has a winning strategy for some constant delay function, which consists of reducing the original problem to a delay-free game with max-regular winning condition. Such a game can be solved by encoding it as an emptiness problem for a certain class of tree automata (so-called WMSO+UP automata) that capture WMSO+UP on infinite trees (a much more expressive logic than the one capturing our winning conditions). Our reduction also yields a doubly-exponential upper bound on the necessary constant delay to win such a game, provided Player $O$ does win for some constant delay function.

It is open whether the doubly-exponential upper bound is tight. The best lower bounds are exponential and hold already for deterministic reachability and safety automata [18, which can easily be transformed into max-automata.

We deliberately skipped the complexity analysis of our algorithm, since the reduction of the delay-free game to an emptiness problem for WMSO+UP automata does most likely not yield tight upper bounds on the complexity. Instead, we propose to investigate (delay-free) games with max-regular winning conditions, a problem that is worthwhile studying on its own, and to find a direct solution algorithm. Currently, the best lower bound on the computational complexity of determining whether Player $O$ wins a delay game with max-regular winning condition for some constant delay function is the ExPTIME-hardness result for games with safety conditions [18].

Also, we showed that constant delay is not sufficient for max-regular conditions by giving a condition $L$ such that Player $O$ wins $\Gamma_{f}(L)$ for some linear $f$, but not for any constant $f$.

Both the lower bound on the necessary lookahead and the one on the computational complexity for safety conditions mentioned above are complemented by matching upper bounds for games with parity conditions [18, i.e., having a parity condition instead of a safety condition has no discernible influence. Stated differently, the complexity of the problems manifests itself in the transition structure of the automaton. Our example from Section 5 shows that this is no longer true for max-regular conditions: having a quantitative acceptance condition requires growing lookahead. 
In ongoing work, we aim to solve delay game with respect to arbitrary delay functions and to determine whether linear delay functions are sufficient to win delay games with max-regular winning conditions.

\section{References}

1. Bojańczyk, M.: Weak MSO with the unbounding quantifier. Theory Comput. Syst. 48(3), 554-576 (2011)

2. Bojańczyk, M.: Weak MSO+U with path quantifiers over infinite trees. In: Esparza et al. 11, pp. 38-49

3. Bojańczyk, M., Gogacz, T., Michalewski, H., Skrzypczak, M.: On the decidability of $\mathrm{MSO}+\mathrm{U}$ on infinite trees. In: Esparza et al. [11, pp. 50-61

4. Bojańczyk, M., Toruńczyk, S.: Weak MSO+U over infinite trees. In: Dürr, C., Wilke, T. (eds.) STACS 2012. LIPIcs, vol. 14, pp. 648-660. Schloss DagstuhlLeibniz-Zentrum fuer Informatik (2012)

5. Büchi, J.R.: On a Decision Method in Restricted Second-Order Arithmetic. In: International Congress on Logic, Methodology, and Philosophy of Science. pp. 111. Stanford University Press (1962)

6. Büchi, J.R., Landweber, L.H.: Solving sequential conditions by finite-state strategies. Trans. Amer. Math. Soc. 138, pp. 295-311 (1969)

7. Carayol, A., Löding, C.: MSO on the infinite binary tree: Choice and order. In: Duparc, J., Henzinger, T.A. (eds.) CSL 2007. LNCS, vol. 4646, pp. 161-176. Springer (2007)

8. Carayol, A., Löding, C.: Uniformization in automata theory. In: Schroeder-Heister, P., Heinzmann, G., Hodges, W., Bour, P.E. (eds.) International Congress of Logic, Methodology and Philosophy of Science. College Publications, London (2012), to appear

9. Chatterjee, K., Henzinger, T.A., Horn, F.: Finitary winning in omega-regular games. ACM Trans. Comput. Log. 11(1) (2009)

10. Emerson, E.A., Jutla, C.S.: Tree automata, mu-calculus and determinacy (extended abstract). In: FOCS 1991. pp. 368-377. IEEE (1991)

11. Esparza, J., Fraigniaud, P., Husfeldt, T., Koutsoupias, E. (eds.): ICALP 2014, Part II, LNCS, vol. 8573. Springer (2014)

12. Faymonville, P., Zimmermann, M.: Parametric linear dynamic logic. In: Peron and Piazza 23, pp. 60-73

13. Fijalkow, N., Zimmermann, M.: Parity and Streett Games with Costs. LMCS 10(2) (2014)

14. Fridman, W., Löding, C., Zimmermann, M.: Degrees of Lookahead in Context-free Infinite Games. In: Bezem, M. (ed.) CSL 2011. LIPIcs, vol. 12, pp. 264-276. Schloss Dagstuhl - Leibniz-Zentrum für Informatik (2011)

15. Grädel, E., Thomas, W., Wilke, T. (eds.): Automata, Logics, and Infinite Games: A Guide to Current Research, LNCS, vol. 2500. Springer (2002)

16. Holtmann, M., Kaiser, L., Thomas, W.: Degrees of lookahead in regular infinite games. LMCS 8(3) (2012)

17. Hosch, F.A., Landweber, L.H.: Finite delay solutions for sequential conditions. In: ICALP 1972. pp. 45-60 (1972)

18. Klein, F., Zimmermann, M.: How Much Lookahead is Needed to Win Infinite Games? ArXiv:1412.3701 (2014) 
19. Kupferman, O., Piterman, N., Vardi, M.Y.: From liveness to promptness. Formal Methods in System Design 34(2), 83-103 (2009)

20. Löding, C., Winter, S.: Synthesis of deterministic top-down tree transducers from automatic tree relations. In: Peron and Piazza [23], pp. 88-101

21. Martin, D.A.: Borel determinacy. Annals of Mathematics 102, 363-371 (1975)

22. Mostowski, A.: Games with forbidden positions. Tech. Rep. 78, University of Gdańsk (1991)

23. Peron, A., Piazza, C. (eds.): GandALF 2014, EPTCS, vol. 161 (2014)

24. Thomas, W., Lescow, H.: Logical specifications of infinite computations. In: de Bakker, J.W., de Roever, W.P., Rozenberg, G. (eds.) A Decade of Concurrency, Reflections and Perspectives, REX School/Symposium. LNCS, vol. 803, pp. 583-621. Springer (1993)

25. Trakhtenbrot, B., Barzdin, I.: Finite Automata; Behavior and Synthesis. Fundamental Studies in Computer Science, V. 1, North-Holland Publishing Company; New York: American Elsevier (1973)

26. Zimmermann, M.: Optimal Bounds in Parametric LTL Games. Theoret. Comput. Sci. 493(0), $30-45$ (2013) 